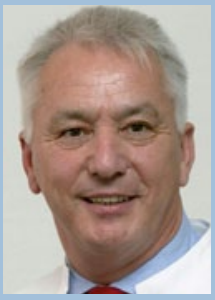

Prof. Dr. med. Curt Diehm Karlsbad, Angiologie

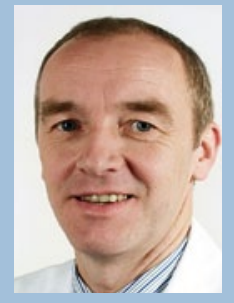

Prof. Dr. med. Joachim Hoyer Marburg, Nephrologie

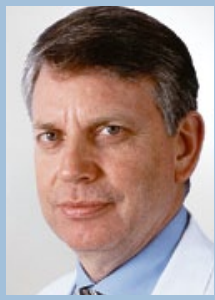

Prof. Dr. med. Gerald Klose Bremen, Lipidologie

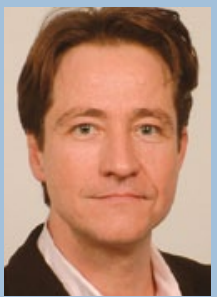

Priv.-Doz. Dr. med. Florian Masuhr Berlin, Neurologie

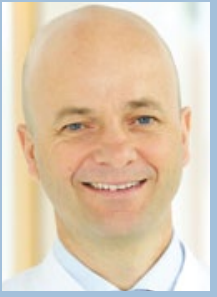

Prof. Dr. med. Georg Nickenig Bonn, Kardiologie

\title{
Perioperative Betablocker-Gabe: Mehr Schaden als Nutzen?
}

In den Leitlinien wird empfohlen, Hochrisikopatienten perioperativ Betablocker zu verabreichen. Einer aktuellen Metaanalyse zufolge steigt dadurch aber das Sterberisiko.

ᄃ ine häufige Frage an Kardiologen vor E nicht kardialen Operationen betrifft die perioperative Medikation. Die 2009 publizierten Leitlinien der Europäischen Gesellschaft für Kardiologie zum perioperativen Management bei nicht kardialen Operationen haben eine KlasseI-Empfehlung für die perioperative Gabe von Betablockern (mit Titrierung der Dosis) bei Hochrisikopatienten (bekannte koronare Herzerkrankung, positiver Ischämienachweis, Hochrisikoeingriff) ausgegeben. Diese Empfehlung basierte auch auf den Ergebnissen der DECREASE-Studien, die Vorteile für die perioperative Betablockergabe zeigten. In letzter Zeit sind jedoch große Beden- ken an der Validität der DECREASEStudien aufgekommen. Zudem haben sowohl eine Metaanalyse aus dem Jahr 2008 wie auch die im gleichen Jahr publizierte POISE-Studie Zweifel am Nutzen der perioperativen Betablockergabe geweckt.

Eine aktuelle Metaanalyse von neun Studien mit insgesamt über $10000 \mathrm{~Pa}$ tienten unter Ausschluss der Ergebnisse der DECREASE-Studien zeigt nun unter einer Betablockergabe sowohl einen relativen Anstieg der perioperativen Mortalität um $27 \%$ sowie einen signifikanten Anstieg von nicht fatalen Schlaganfällen (73\%) und Hypotensionen (51\%; s. Abb.).
Bouri $S$ et al. Meta-analysis of secure randomised controlled trials of $\beta$-blockade to prevent perioperative death in non-cardiac surgery. Heart doi:10.1136/heartjnl-2013-304262

Kommentar: Die Ergebnisse dieser Metaanalyse stellen das bislang praktizierte und durch Leitlinien empfohlene Vorgehen zur perioperativen Gabe von Betablockern infrage. Welche Konsequenzen sollten daraus gezogen werden? Meines Erachtens sind mehrere Aspekte zu beachten:

1. Eine gründliche präoperative, leitlinienkonforme Risikostratifizierung ist erforderlich.

2. Eine bestehende Betablocker-Medikation sollte präoperativ nicht abgesetzt werden. 3. Falls eine Betablocker-Therapie präoperativ initiiert werden sollte, sind beta-1-selektive Betablocker zu bevorzugen und in ihrer Dosis sorgsam zu titrieren.

Es bleibt abzuwarten, ob und wie die Leitlinien-Komitees diese Unklarheiten in einer klinisch so wichtigen und häufigen Fragestellung auflösen werden.

Prof. Dr. med. Peter W. Radke,

Schön Klinik Neustadt

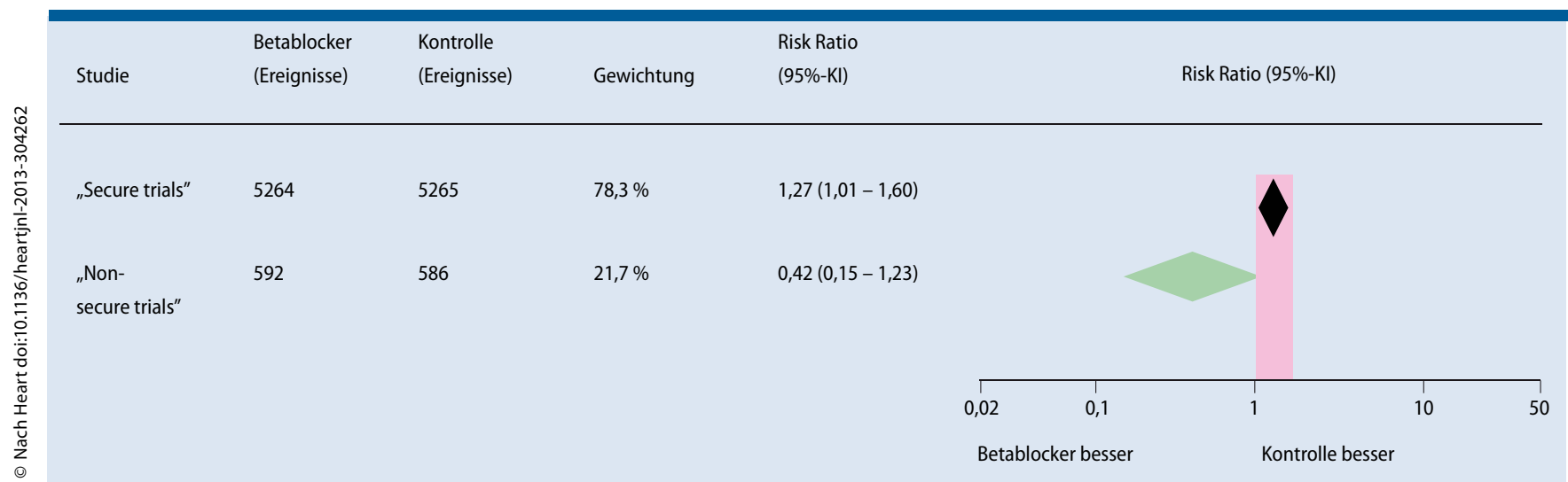

Effekte der perioperativen Betablockergabe unter Ausschluss der DECREASE-Studien („secure trials“) im Vergleich zu den DECREASEStudien („non secure trials“). 